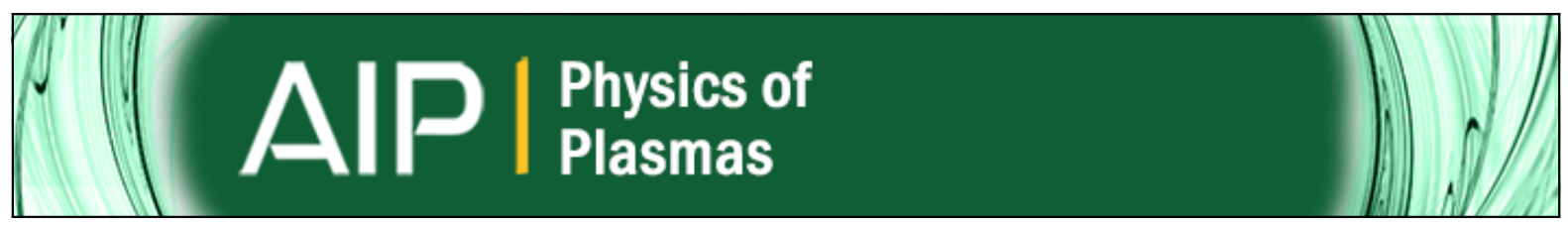

Ion finite Larmor radius effects on the interchange instability in an open system

I. Katanuma, S. Sato, Y. Okuyama, S. Kato, and R. Kubota

Citation: Physics of Plasmas (1994-present) 20, 112107 (2013); doi: 10.1063/1.4829682

View online: http://dx.doi.org/10.1063/1.4829682

View Table of Contents: http://scitation.aip.org/content/aip/journal/pop/20/11?ver=pdfcov

Published by the AIP Publishing

AlP Re-register for Table of Content Alerts

Create a profile.

Sign up today! 


\title{
Ion finite Larmor radius effects on the interchange instability in an open system
}

\author{
I. Katanuma, a) S. Sato, Y. Okuyama, S. Kato, and R. Kubota \\ Plasma Research Center, University of Tsukuba, Tsukuba, Ibaraki 305-8577, Japan
}

(Received 10 September 2013; accepted 24 October 2013; published online 14 November 2013)

\begin{abstract}
A particle simulation of an interchange instability was performed by taking into account the ion finite Larmor radius (FLR) effects. It is found that the interchange instability with large FLR grows in two phases, that is, linearly growing phase and the nonlinear phase subsequent to the linear phase, where the instability grows exponentially in both phases. The linear growth rates observed in the simulation agree well with the theoretical calculation. The effects of FLR are usually taken in the fluid simulation through the gyroviscosity, the effects of which are verified in the particle simulation with large FLR regime. The gyroviscous cancellation phenomenon observed in the particle simulation causes the drifts in the direction of ion diamagnetic drifts. (C) 2013 Author(s). All article content, except where otherwise noted, is licensed under a Creative Commons Attribution 3.0 Unported License. [http://dx.doi.org/10.1063/1.4829682]
\end{abstract}

\section{INTRODUCTION}

The interchange instability is perhaps the most fundamental and basic magnetohydrodynamic (MHD) instability for magnetically confined plasma. There are some methods to stabilize the interchange instability. One is to make use of the line tying effects. ${ }^{1,2}$ The magnetic surfaces created in the torus, ${ }^{3}$ for example, stabilize the interchange instability with the rotation of the magnetic field lines lying on the magnetic surface, which is the results of line tying effects.

The ion finite Larmor radius (FLR) effects are also expected to stabilize the interchange instability. The first work of the FLR effects on an interchange instability was seen in Ref. 4, where Rosenbluth, Krall, and Rostoker have found the stability effects of FLR with the help of Vlasov equation. Roberts and Taylor derived the same stability condition as Ref. 4 by using the extended MHD equations. ${ }^{5}$ Here, they used the generalized Ohm's law and they included the viscosity in the equation of motion. The extended MHD equations have been widely used to study interchange instability, because they are able to be applied to the complicated magnetic confined closed systems. ${ }^{6,7}$ The FLR effects on an interchange instability are also important in an open system such as a tandem mirror. ${ }^{8-12}$

The generalized Ohm's law and gyroviscosity in the extended MHD equation were derived theoretically. ${ }^{5,13}$ Recently, it has been reported that the complete FLR stabilization of the interchange mode may not be attainable by the gyroviscosity or generalized Ohm's law alone in the frame work of extended MHD. ${ }^{7}$ The stability boundary of an interchange instability with FLR was determined by the kinetic analysis. ${ }^{4,14}$ Thus, it is worth verifying with the help of a particle simulation that the interchange instability can be really stabilized by FLR effects completely.

The traditional electrostatic particle-in-cell code (explicit PIC code) uses the equation of ion and electron

\footnotetext{
${ }^{a)}$ Electronic address: katanuma@prc.tsukuba.ac.jp
}

motions with the Poisson equation. ${ }^{15,16}$ The mesh interval $\Delta$ and the time step $\Delta t$ have to be taken smaller than the Debye length $\lambda_{D e}$ and the inverse of the plasma oscillation $\omega_{p e}^{-1}$ and electron cyclotron frequency $\Omega_{c e}^{-1}$, respectively, because all of the electrostatic oscillations are included in the explicit code.

The interchange instability by using the explicit PIC code was carried out by Goede, Humanic, and Dawson, ${ }^{17}$ where two-dimensional spatial $64 \times 64$ grid was used. In order to follow such a slow time scale of interchange instability, they used the very small ion/electron mass ratio $M_{i} / M_{e}=1$. The linear growth rate of an interchange instability observed in the simulation did not compare well with the growth rate derived from the Vlasov equation in a local approximation, although it showed the stabilization due to FLR effects. Recently, the interchange instabilities mainly in the magnetotail are simulated by the electromagnetic explicit PIC code. ${ }^{18,19}$ However, the comparison of the interchange instabilities itself observed in the simulation with those derived theoretically has not been made.

The increases of the time step $\Delta t$ and the grid interval $\Delta$ enable to simulate a low frequency phenomena in the plasma on a large scale. The implicit time integration scheme has a potential to increase $\Delta t$ and $\Delta$ keeping the numerical stability of the simulation. Cohen et al. have described the implicit plasma simulation algorithm and analyzed for onedimensional case. ${ }^{20-22}$ Watanabe et al. have described the implicit algorithm and applied to the two-dimensional plasma with external magnetic field, where ion and electron cross-field motions are assumed only $\boldsymbol{E} \times \boldsymbol{B}$ drifts. ${ }^{23}$ Barnes et al. have developed the implicit algorithm which can be able to applied to the two-dimensional plasma with the external magnetic field directly, and they demonstrated the simulation on an interchange instability (without FLR). ${ }^{24}$

This paper uses the uniform gravitational field $\mathbf{g}=\mathrm{g} \hat{e}_{x}$ shown in Fig. 1, where $\hat{e}_{x}$ is the unit vector along $x$-axis. Here, the centrifugal force due to the non-zero magnetic field line curvature is replaced by the gravitational force. The 


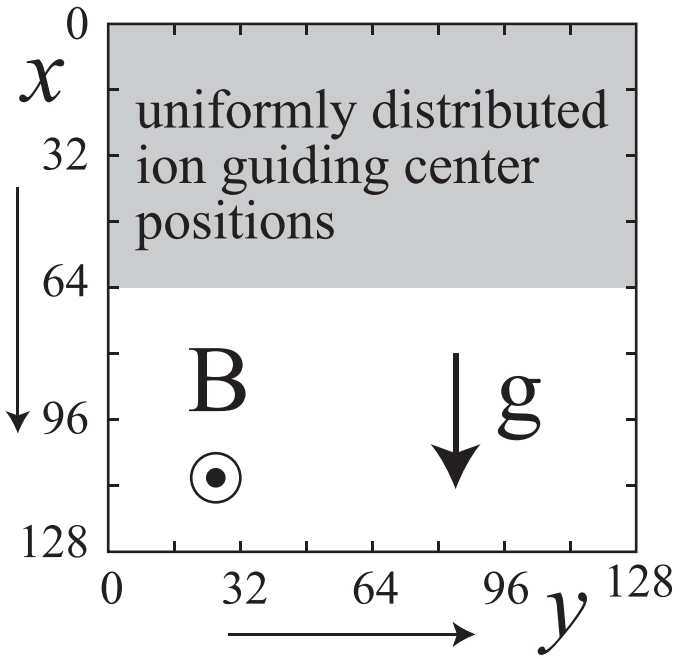

FIG. 1. Initial ion guiding center positions, gravitational acceleration vector (g), and external magnetic field. Vertical (horizontal) axis is the $x$ axis $(y$ axis) with the scale in mesh numbers.

uniform external magnetic field $\mathbf{B}=\mathrm{B} \hat{e}_{z}$ is applied along $z$-axis. The FLR effects on the interchange instability are investigated in the geometry of Fig. 1. The PIC code used in this paper has adopted the implicit algorithm by Barnes et al. ${ }^{24}$

\section{LINEAR GROWTH RATES OF AN INTERCHANGE INSTABILITY WITH FLR}

Rosenbluth and Simon derived the dispersion relation of an interchange instability with the FLR effects ${ }^{14}$

$$
\begin{aligned}
& \frac{\mathrm{d}}{\mathrm{d} x}\left(T \frac{\mathrm{d} \psi}{\mathrm{d} x}\right)-k_{y}^{2}\left(T-\mathrm{g} \frac{\mathrm{d} \rho}{\mathrm{d} x}\right) \psi=0 \quad \text { where } \\
& \psi=\frac{E_{y}}{\left(\omega-c k_{y} E_{0} / B\right)},
\end{aligned}
$$

where $\omega$ is the wave frequency, $k_{y}$ is the wave number, $E_{y}$ is the perturbed electric field of the interchange instability, and $c$ is the light speed. The steady state electric field $E_{0}$, gravitational acceleration $g$ and equilibrium density gradient $\mathrm{d} \rho / \mathrm{d} x$ are assumed to be a function of $x$ and their vectors are in the $x$ direction. The uniform external magnetic field $\boldsymbol{B}$ is assumed to be applied in the $z$ direction. The coefficients $T$ and $S$ in Eq. (1) are defined as

$$
\begin{aligned}
T & \equiv\left(\omega-c k_{y} E_{0} / B\right)^{2} \rho S, \\
S & \equiv 1+\frac{\Omega_{c i}^{2}}{\omega_{p i}^{2}}+\frac{k_{y}}{\rho \Omega_{c i}\left(\omega-c k_{y} E_{0} / B\right)} \frac{\mathrm{d} P}{\mathrm{~d} x},
\end{aligned}
$$

where $\omega_{p i} \equiv \sqrt{4 \pi n_{i} e^{2} / M_{i}}$ is the ion plasma frequency, $\Omega_{c i} \equiv e B / M_{i} c$ is the ion cyclotron frequency, $e$ is the unit charge, $M_{i}$ is the ion mass, and $n_{i}$ is the ion number density. The mass density $\rho$ and pressure $P$ of ions are given as

$$
P=\frac{M_{i}}{2} \int v^{2} f_{i} \mathrm{~d} \boldsymbol{v}, \quad \rho=M_{i} \int f_{i} \mathrm{~d} \boldsymbol{v} .
$$

Here, $f_{i}$ is the ion distribution function in the equilibrium state as

$$
f_{i}(X, \boldsymbol{v})=n_{g}(X)\left(\frac{M_{i}}{2 \pi T_{i}}\right)^{3 / 2} \exp \left\{-\frac{M_{i} \boldsymbol{v}^{2}}{2 T_{i}}\right\} .
$$

The distribution in the equilibrium state should be a function of constant of motion, so that $X$ is a canonical momentum in the $y$ direction which is the same as the guiding center position in the case of uniform external magnetic field, i.e., $X=x+v_{y} / \Omega_{c i}$, where $x$ is the real position of ion in the $x$ direction. Henceforth, the ion guiding center density profile $n_{g}(X)$ is assumed to be

$$
n_{g}(X)=n_{0}\left[1-\theta\left(X-L_{H}\right)\right],
$$

where $\theta(x)$ is the Heaviside step function $[\theta(x)=0$ for $x<0$ and $\theta(x)=1$ for $x>0$ ]. The guiding center density $n_{g}(X)$ of Eq. (5) lead to the real density $n_{i}(x)$ of

$$
n_{i}(x)= \begin{cases}\frac{n_{0}}{2}\left(1+\operatorname{erf}\left\{-\left(x-L_{H}\right) /\left(\sqrt{2} \rho_{i}\right)\right\}\right) & \text { for } x<L_{H} \\ \frac{n_{0}}{2}\left(1-\operatorname{erf}\left\{\left(x-L_{H}\right) /\left(\sqrt{2} \rho_{i}\right)\right\}\right) & \text { for } x>L_{H},\end{cases}
$$

where $\rho_{i} \equiv \sqrt{T_{i} / M_{i}} / \Omega_{c i}$ is the ion Larmor radius. The error function $\operatorname{erf}(x)$ is defined as

$$
\operatorname{erf}\{x\} \equiv \frac{2}{\sqrt{\pi}} \int_{0}^{x} \exp \left\{-t^{2}\right\} \mathrm{d} t .
$$

Figure 2 plots the linear growth rates of an interchange instability with FLR in the case of $k_{y} \mathrm{~g} / \Omega_{c i}^{2}=10^{-4}$, which
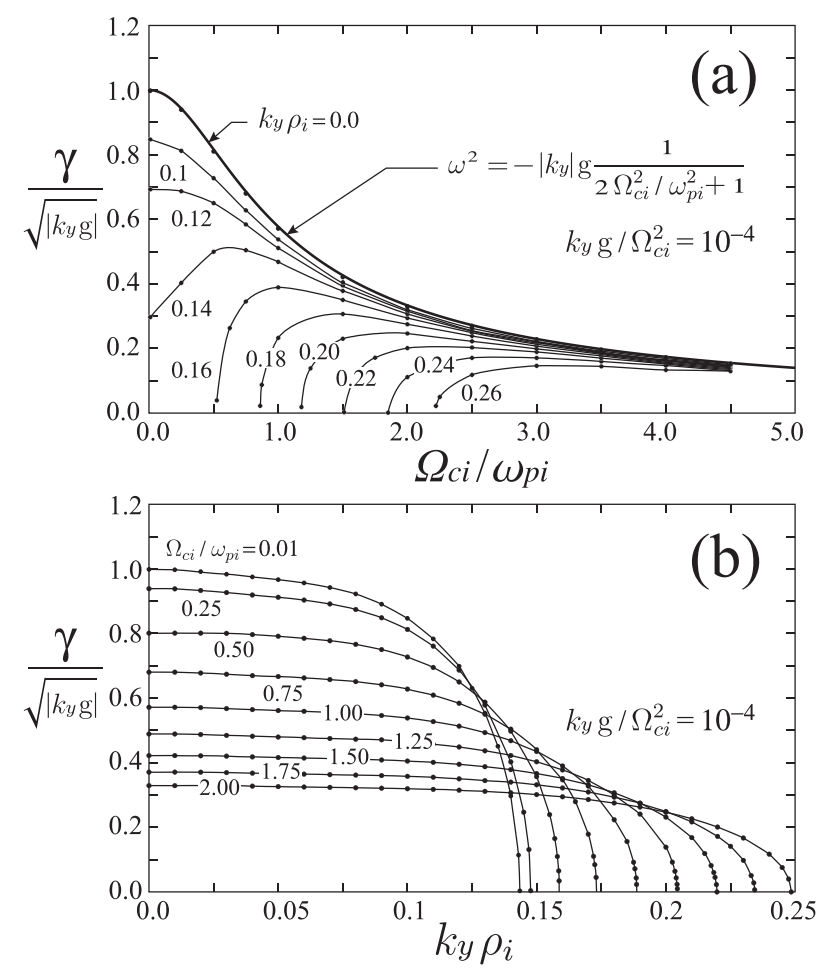

FIG. 2. Dispersion relation of the interchange instabilities with FLR. (a) is the linear growth rates $\gamma$ as a function of $\Omega_{c i} / \omega_{p i}$, where each number in (a) denotes the magnitude of $k_{y} \rho_{i}$. (b) is the linear growth rates as a function $k_{y} \rho_{i}$, where each number in (b) denotes the magnitude of $\Omega_{c i} / \omega_{p i}$. 
was obtained by solving Eq. (1) in the range of $0 \leq k_{y} x \leq 2 \pi$ with the boundary condition that $\psi=0$ at $k_{y} x=0$ and $\psi=0$ at $k_{y} x=2 \pi$ and $k_{y} L_{H}=\pi$. The vertical axis $\gamma$ in Fig. 2 is the linear growth rate of the interchange instability, i.e., $\omega=\omega_{r}+\mathrm{i} \gamma$, where $\mathrm{i} \equiv \sqrt{-1}$. The linear dispersion relation in the geometry of Fig. 1 for $k_{y} \rho_{i}=0$ is given by ${ }^{25}$

$$
\omega^{2}=-\left|k_{y}\right| \mathrm{g} \frac{1}{2 \Omega_{i}^{2} / \omega_{p i}^{2}+1},
$$

which is plotted in Fig. 2(a) in case of $k_{y} \rho_{i}=0$. Figure 2 indicates that the interchange instability is stabilized more strongly by FLR for the smaller $\Omega_{c i} / \omega_{p i}$ and larger $k_{y} \rho_{i}$.

\section{ELECTROSTATIC PIC CODE}

The electrostatic PIC code is used in order to research the stabilization effects of FLR on an interchange instability. The PIC code used in this paper adopts the implicit algorithm, ${ }^{1,2,24}$ so as to remove the unnecessary high frequency electrostatic oscillations such as the electron cyclotron waves.

The equation of motion makes use of the modified leapfrog differential scheme

$$
\begin{aligned}
\boldsymbol{x}_{j}^{n+1} & =\boldsymbol{x}_{j}^{n}+\boldsymbol{v}_{j}^{n+1 / 2} \Delta t, \\
\boldsymbol{v}_{j}^{n+1 / 2} & =\boldsymbol{v}_{j}^{n-1 / 2}-\frac{e_{\alpha}}{M_{\alpha}} \nabla \bar{\phi}^{n} \Delta t+\frac{1}{c} \boldsymbol{v}_{j}^{n+\gamma_{0}} \times \boldsymbol{\Omega}_{c \alpha} \Delta t+\mathbf{g} \Delta t,
\end{aligned}
$$

where the superscript $n$ means the time step $n \Delta t$ and the subscript $\alpha$ indicates the particle species.

The electrostatic potential $\bar{\phi}^{n}$ is solved by the following equations:

$$
\begin{aligned}
& \bar{\phi}^{n}=\frac{2}{5}\left(\phi^{n+1}+2 \bar{\phi}^{n-1}-\frac{1}{2} \bar{\phi}^{n-2}\right), \\
& \nabla^{2} \phi^{n+1}=-4 \pi e\left(n_{i}^{n+1}-n_{e}^{n+1}\right),
\end{aligned}
$$

where the second equation in Eq. (10) is the Poisson equation.

The velocity $\boldsymbol{v}_{j}^{n+\gamma_{0}}$ in the right hand side of Eq. (9) is defined by

$$
\boldsymbol{v}_{j}^{n+\gamma_{0}}=\left(\frac{1}{2}+\gamma_{0}\right) \boldsymbol{v}_{j}^{n+1 / 2}+\left(\frac{1}{2}-\gamma_{0}\right) \boldsymbol{v}_{j}^{n-1 / 2} .
$$

If $\gamma_{0}=0$ and $\phi^{n}$ instead of $\bar{\phi}^{n}$ are used, Eq. (9) becomes the normal centered leap-frog scheme with the second order accuracy of time step $\Delta t$ and is numerically unstable if $\left|\boldsymbol{\Omega}_{c \alpha}\right| \Delta t>1$. The detailed algorithm of the implicit PIC code is given in Ref. 24.

In this paper, $\gamma_{0}=0.1$ has been adopted for electrons and the time step is chosen $\omega_{p e} \Delta t=2$ for the simulation of an interchange instability, where $\omega_{p e} \equiv \sqrt{4 \pi n_{e} e^{2} / M_{e}}$ is the electron plasma frequency. However, $\gamma_{0}=0$ is chosen for ions so that the ion Larmor motion is followed with the second order accuracy of time step $\Delta t$.

\section{SIMULATION RESULTS}

The code uses the two-dimensional $128 \times 128$ spatial meshes in $x-y$ and three velocity components $v_{x}, v_{y}$, and $v_{z}$. The $(4 \times 128)^{2}=262,144$ ions, and electrons each are included in the simulation. The geometry used in the simulation and analysis is plotted in Fig. 1. The ion guiding centers are uniformly distributed in the region $x<64 \Delta$, where $\Delta$ is the spatial mesh interval, at $t=0$ and electron real positions are distributed at each ion real position. The uniform magnetic field $B$ is applied in the $z$ direction and gravitational acceleration $\mathbf{g}$ is in the $x$ direction $\left(\mathbf{g}=\mathrm{g} \hat{e}_{x}\right)$, as shown in Fig. 1. Henceforth, $\omega_{p i} \equiv \sqrt{4 \pi\left\langle n_{i}\right\rangle e^{2} / M_{i}}$ and $\omega_{p e} \equiv$ $\sqrt{4 \pi\left\langle n_{i}\right\rangle e^{2} / M_{e}}$ are used as ion and electron plasma frequencies, where $\left\langle n_{i}\right\rangle=n_{0} / 2$ is the ion (electron) density averaged over the entire simulation space.

The interchange instability is observed in the simulation because the geometry in Fig. 1 is unstable to the interchange modes. Figure 3 plots the linear growth rates of interchange instability with $(1,1)$ mode measured in the linearly growing phase of the simulation. Here the $(1,1)$ mode means the one with the wave numbers $k_{y}=2 \pi / 128 \Delta$ and $k_{x}=2 \pi / 128 \Delta$. The solid circles in Fig. 3 are the results without FLR, where $\gamma_{0}=0.1$ has been chosen for ions in Eqs. (9) and (11). The solid line arrowed by a symbol $(A)$ is the theoretically calculated linear growth rate Eq. (8).

The solid squares in Fig. 3 are the simulation results with FLR, where $\gamma_{0}=0$ is set for ions in Eqs. (9) and (11). The solid line arrowed by a symbol B is the theoretically calculated linear growth rate in case of $k_{y} \rho_{i}=0.16$ in Fig. 2(a), where $k_{y} \rho_{i} /\left(k_{y} \mathrm{~g} / \Omega_{c i}^{2}\right)^{1 / 4}=1.6$. The simulation parameters are that $k_{y}\left\langle\rho_{i}\right\rangle=2.845 \times 10^{-1}, \Omega_{c i} / \omega_{p e}=2.2155 \times 10^{-3}$, $k_{y} \mathrm{~g} / \Omega_{c i}^{2}=1.0 \times 10^{-3}$, where $k_{y}=2 \pi / 128 \Delta$ and $\left\langle\rho_{i}\right\rangle$ $=\sqrt{T_{i} / M_{i}} / \Omega_{c i}$, that is, $k_{y}\left\langle\rho_{i}\right\rangle /\left(k_{y} \mathrm{~g} / \Omega_{c i}^{2}\right)^{1 / 4}=1.6$. Note that all quantities in Eqs. (1) and (2) are normalized as

$$
\frac{\omega}{\sqrt{k_{y}|\mathrm{~g}|}}, \quad \frac{\omega_{E}}{\sqrt{k_{y}|\mathrm{~g}|}}, \quad \frac{k_{y} \rho_{i}}{\left(k_{y}|\mathrm{~g}| / \Omega_{c i}^{2}\right)^{1 / 4}}, \quad \frac{\omega_{p i}}{\Omega_{c i}},
$$

where $\omega_{E} \equiv k_{y} c E_{0} / B$, although $E_{0}=0$ is assumed throughout this paper. The solid squares in Fig. 3 were obtained by

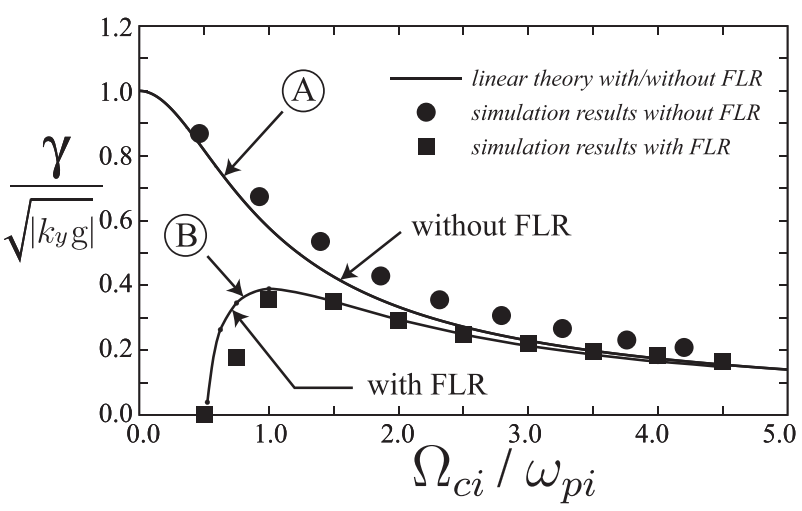

FIG. 3. Dispersion relation of the interchange instabilities in theory and simulation. The solid circles are the growth rates of the $(1,1)$ mode measured in the linearly growing phase of the interchange instability in the simulation without FLR, while the solid squares are the ones with FLR. The solid lines arrowed by the symbols $(A)$ and $(B)$ are the theoretical linear growth rates without/with FLR, respectively. 
changing the ion mass $M_{i}$ and the initial ion temperature $T_{i}$ in the simulation. The agreements between theory and simulation are good in both cases with and without FLR in Fig. 3. It is found that the flute instability is stabilized at $\Omega_{c i} / \omega_{p i} \simeq$ 0.5 in Fig. 3.

Figure 4 plots the simulation results with parameters that $M_{i} / M_{e}=1800, k_{y} \mathrm{~g} / \Omega_{c i}^{2}=9.94 \times 10^{-4}, \Omega_{c i} / \omega_{p i}=0.943$, $\Omega_{c e} / \omega_{p e}=40$. The ion thermal Larmor radius $\left\langle\rho_{i}\right\rangle$ in Fig. 4 is normalized by the spatial mesh interval $\Delta$. The solid circles which are the linear growth rate observed in the simulation are obtained by changing initial ion temperature $T_{i}$. The solid line in Fig. 4 is obtained by solving Eq. (1) with $\Omega_{c i} / \omega_{p i}=0.943$. The agreements between the linear growth rates obtained analytically and by using simulation are good. There is the apparent systematic (but small) discrepancy observed in Fig. 4 at small values of the ion Larmor radius $\left\langle\rho_{i}\right\rangle<2$. The linear growth rates (solid circles) in Fig. 4 have been measured from the wave form of field energy as shown in Fig. 5. Thus, the discrepancy is beyond the measuring error, but we do not know the reason. This systematic discrepancy can be seen in Fig. 3 at $\left\langle\rho_{i}\right\rangle=0$, which is consistent with those in Fig. 4.

The time evolutions of field energy $\left|\phi_{k}\right|^{2}$ of $(1,1)$ mode are plotted in Fig. 5. The dotted straight lines in the figure were used to obtain the linear growth rates (solid circles) in Fig. 4. Here, each origin of field energy has been shifted upward (or downward) at the amount of arbitral magnitudes in the figure, so that the relative magnitudes of $\left|\phi_{k}\right|^{2}$ with different $\left\langle\rho_{i}\right\rangle$ are meaningless in Fig. 5.

Figure 6 plots the ion and electron real positions at $\omega_{\text {pet }}=11000$, which is the time when the interchange instability enters the nonlinear growing phase from the linear phase as is seen in Fig. 5. The ion real positions are plotted in the upper figures and the electrons are in the lower figures, respectively. It is found that the quasi-neutral condition is satisfied very much in all cases of $\left\langle\rho_{i}\right\rangle$. Here, Fig. 6(a) is the simulation result with $\left\langle\rho_{i}\right\rangle=0.5$, Fig. 6 (b) is $\left\langle\rho_{i}\right\rangle=1.5$, Fig. 6(c) is $\left\langle\rho_{i}\right\rangle=3.0$, and Fig. 6(d) is $\left\langle\rho_{i}\right\rangle=6.0$, respectively. When the thermal ion Larmor radius $\left\langle\rho_{i}\right\rangle$, where normalized by a spatial mesh interval $\Delta$, is small, there can be seen many interchange instabilities with high mode numbers, while in the case of large $\left\langle\rho_{i}\right\rangle$ an interchange instability with

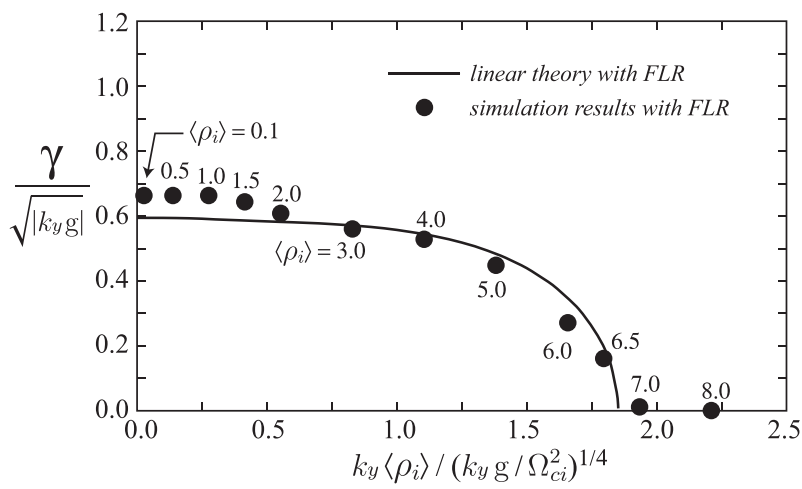

FIG. 4. Dispersion relation of the interchange instabilities in theory and simulation. The solid circles are the growth rates of the $(1,1)$ mode measured in the linearly growing phase of the interchange instability in the simulation with FLR. The solid line is the theoretical linear growth rates with FLR.
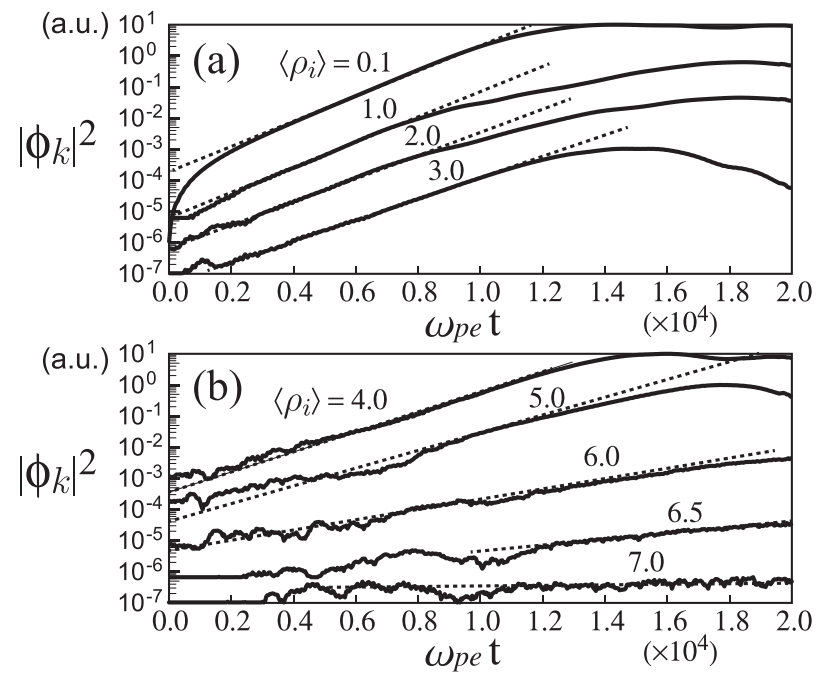

FIG. 5. Time evolution of field energy $\left|\phi_{k}\right|^{2}$ with $(1,1)$ mode. The dashed straight lines in the figure are drawn in order to determine the linear growth rates in the simulation. The numbers in the figure denote the magnitudes of thermal ion Larmor radius $\left\langle\rho_{i}\right\rangle$, which are normalized by the mesh interval $\Delta$. Here, each origin of field energy $\left|\phi_{k}\right|^{2}$ has been shifted upward (or downward) in order to display the growing phase clearly, so that the relative magnitudes of $\left|\phi_{k}\right|^{2}$ with different $\left\langle\rho_{i}\right\rangle$ are meaningless in Fig. 5.

only the lowest $k_{y}$ is seen. The interchange instability is almost stabilized by FLR for $\left\langle\rho_{i}\right\rangle=6.0$. The ions begin to behave like a viscous fluid in the presence of the magnetic viscosity in Figs. 6(c) and 6(d). Equation (8), which is the linear growth rate in case $\rho_{i}=0$, indicates that the linear growth rate is $\gamma \propto \sqrt{\left|k_{y}\right|}$. In the simulation with $\left\langle\rho_{i}\right\rangle \neq 0$, ions and electrons have the initial Maxwellian velocities, so that the thermal fluctuations excite the interchange modes with various $k_{y}$. In the case of $\left\langle\rho_{i}\right\rangle \lesssim 2$ the interchange mode with high $k_{y}$ are more unstable than that with low $k_{y}$, so that many saturated high $k_{y}$ interchange instabilities with mushroom-shaped front ${ }^{1,6}$ are observed in Figs. 6(a) and 6(b). On the other hand, the high $k_{y}$ interchange modes are stabilized by the FLR effects in case of $\left\langle\rho_{i}\right\rangle \geqslant 2$ and so the only interchange instability with the lowest $k_{y}$ is observed in Figs. 6(c) and 6(d).

Figure 7 plots the time evolution of $\left|\phi_{k}\right|^{2}$ of $(1,1)$ mode in the case of $k_{y} \mathrm{~g} / \Omega_{c i}^{2}=10^{-3}, \Omega_{c i} / \omega_{p i}=1.0,\left\langle\rho_{i}\right\rangle \simeq 5.796$. It is found that an interchange instability grows with different growth rates in the linear phase (solid straight line) and in the nonlinear phase (dotted broken straight line). Here the growth rate (solid straight line) in the linear growing phase has been calculated by the linear theory Eq. (1). The growth rates in the nonlinear phase are slower than those in the linear phase in Figs. 5 and 7.

As is seen in Fig. 8, in the linear phase $\left(\omega_{p e} t=4000\right.$ $\sim 10000$ in Fig. 7) the boundary between ions and vacuum has a sinusoidal shape. However, in the nonlinear phase, the shape of the boundary is distorted from the sinusoidal curve greatly. Here, Figs. 8(a)-8(d) plot the equi-contour lines of ion density at $\omega_{p e} t=8000,11000,14000$, and 17000 , respectively. Figures 8 and 7 are the results of the same simulation. As is seen in Fig. 7, the time $7000 \leqq \omega_{p e} t \lesssim 12000$ is the linearly growing phase of the interchange instability, so that the equi-contour lines (boundary between plasma and 


\section{$\omega_{p e} \mathrm{t}=11000$}

(a) $\left\langle\rho_{i}\right\rangle=0.5$
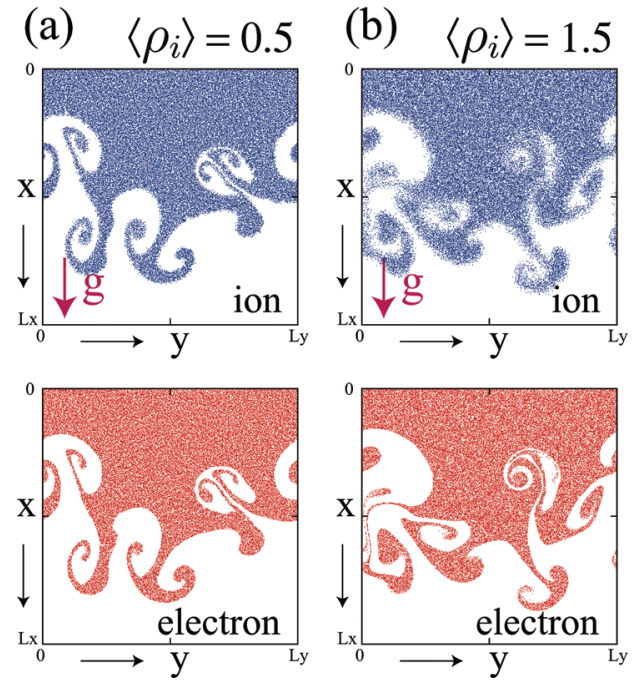

(c) $\left\langle\rho_{i}\right\rangle=3.0$

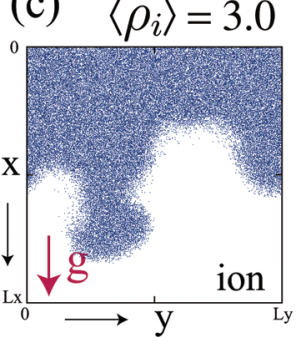

(d) $\left\langle\rho_{i}\right\rangle=6.0$
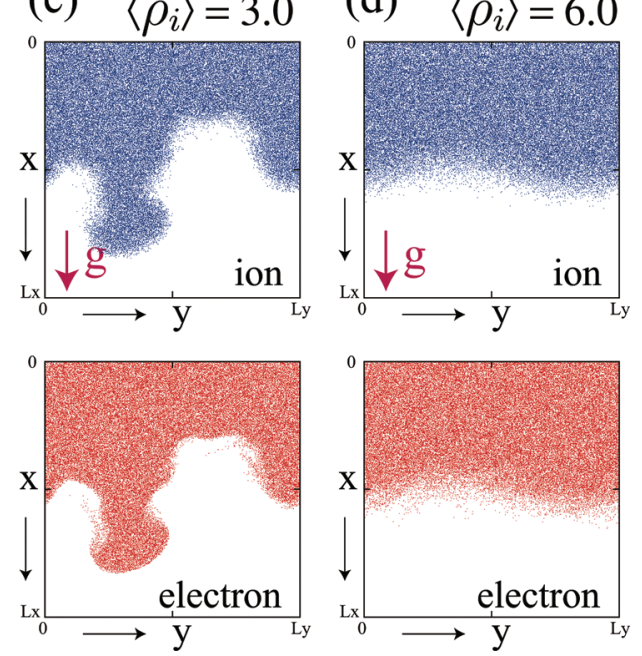

FIG. 6. Real space positions of ion (in the upper figures) and electron (in the lower figures) at $\omega_{\text {pet }}=11000$. Here, (a) is the simulation result with $\left\langle\rho_{i}\right\rangle=0.5$, (b) is $\left\langle\rho_{i}\right\rangle=1.5$, (c) is $\left\langle\rho_{i}\right\rangle=3.0$, and (d) is $\left\langle\rho_{i}\right\rangle=6.0$.

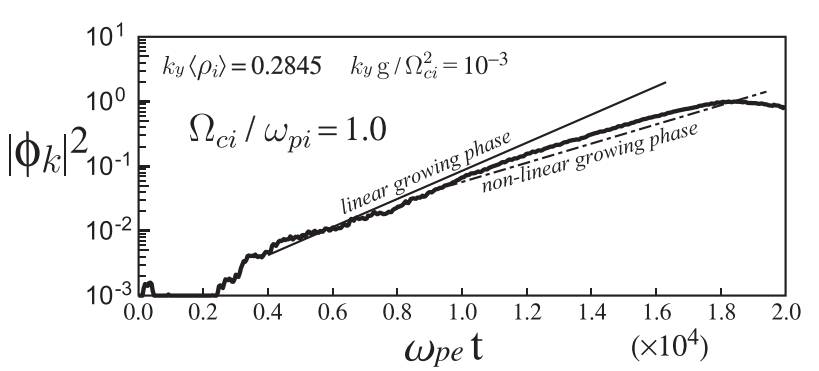

FIG. 7. The time evolution of field energy $\left|\phi_{k}\right|^{2}$ with $(1,1)$ mode. The solid straight line denotes the linear growth rate which was calculated analytically by using Eq. (1). The dashed straight line is drawn to fit the growth rate to the wave form in the nonlinear growing phase of $\left|\phi_{k}\right|^{2}$. Here, the figure is the simulation result in the case $k_{y} \mathrm{~g} / \Omega_{c i}^{2}=10^{-3}, \Omega_{c i} / \omega_{p i}=1.0$, $\left\langle\rho_{i}\right\rangle \simeq 5.796$.

vacuum) in Figs. 8(a) and 8(b) can be approximate sinusoidal function well. On the other hand the time $12000 \lessgtr \omega_{p e} t$ $\lesssim 18000$ is the nonlinearly growing phase of the instability, so that the equi-contour lines (boundary between plasma and vacuum) in Figs. 8(c) and 8(d) deviate from the sinusoidal function greatly.

The remarkable feature in Fig. 8(d) is that the front of the flute instability drifts in the $-y$ direction, which is the ion diamagnetic direction. It is known that this drifts result from the gyroviscosity. The equation of motion in the extended MHD is written to be $\mathrm{e}^{5,7}$

$$
\rho\left(\frac{\partial \boldsymbol{u}}{\partial t}+\boldsymbol{u} \cdot \nabla \boldsymbol{u}\right)=-\nabla p+\frac{1}{c} \boldsymbol{j} \times \boldsymbol{B}+\rho \mathbf{g}-\nabla \cdot \boldsymbol{\Pi}_{i} .
$$

The last term $\nabla \cdot \boldsymbol{\Pi}_{i}$ is the viscosity term containing the twofluid effects and ion gyroviscosity effects. Frequently, the gyroviscous stress is approximated by ${ }^{6,26}$

$$
\nabla \cdot \boldsymbol{\Pi}_{i} \simeq-\rho \boldsymbol{u}_{*} \cdot \nabla \boldsymbol{u} .
$$

Here, $\boldsymbol{u}_{*}$ is often assumed to be the ion diamagnetic drift velocity in the uniform magnetic field, i.e.,

$$
\boldsymbol{u}_{*}=\frac{-c \nabla p \times \boldsymbol{B}}{n_{i} e B^{2}} .
$$

Moving the gyroviscosity term to the left hand in Eq. (13) in the approximation of Eq. (14) yields

$$
\rho\left(\frac{\partial \boldsymbol{u}}{\partial t}+\boldsymbol{u} \cdot \nabla \boldsymbol{u}\right)+\nabla \cdot \boldsymbol{\Pi}_{i}=\rho\left(\frac{\partial \boldsymbol{u}}{\partial t}+\left(\boldsymbol{u}-\boldsymbol{u}_{*}\right) \cdot \nabla \boldsymbol{u}\right),
$$

$$
\text { ion density } \quad k_{y}\left\langle\rho_{i}\right\rangle=0.2845 \quad k_{y} \mathrm{~g} / \Omega_{c i}^{2}=10^{-3} \quad \Omega_{c i} / \omega_{p i}=1.0
$$

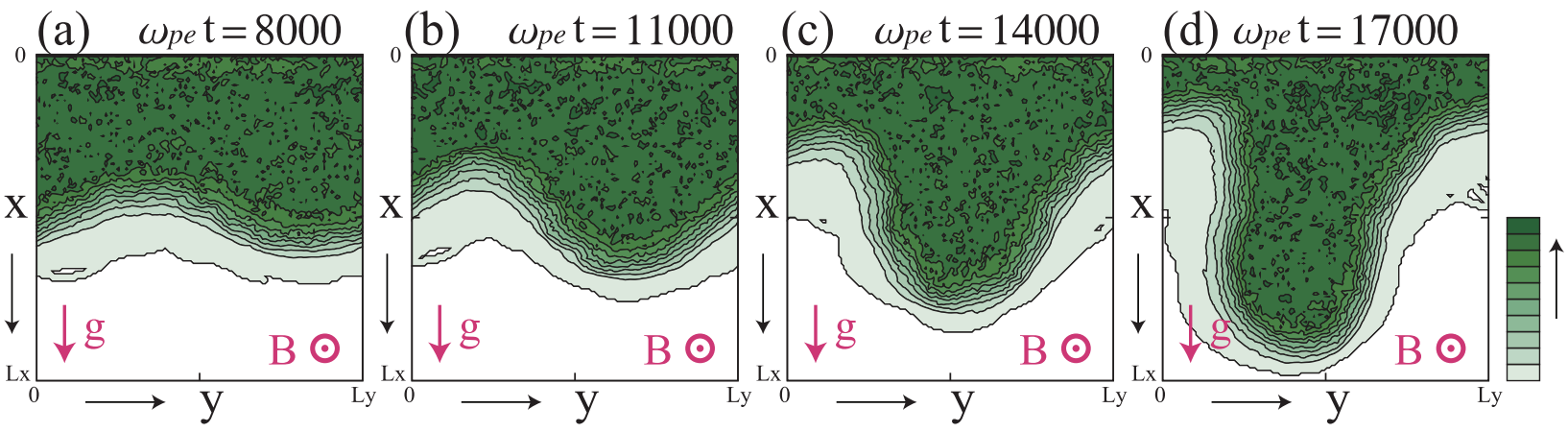

FIG. 8. Contour plots of ion number density. Here, the figure is the simulation result in the case $k_{y} \mathrm{~g} / \Omega_{c i}^{2}=10^{-3}, \Omega_{c i} / \omega_{p i}=1.0,\left\langle\rho_{i}\right\rangle \simeq 5.796$. (a) is the contour plot of the ion density at $\omega_{p e} t=8000$, (b) is at $\omega_{p e} t=11000$, (c) is $\omega_{p e} t=14000$, and (d) is $\omega_{p e} t=17000$, respectively. 
The simulation has not solve the time evolution of the magnetic field because the electrostatic PIC code are used. That is, the uniform magnetic field remains until the end of the simulation. The equilibrium ion drift velocity, therefore, comes from the gravitational drift which is given by $\boldsymbol{u}_{\mathrm{g}}=$ $c M_{i} \mathbf{g} \times \boldsymbol{B} / q B^{2}=-4.5 \times 10^{-4} \omega_{p e} \Delta \hat{e}_{y}$ in the parameters used to obtain Fig. 8. Here, Fig. 8 reveals that the interchange instability drifts in the $-y$ direction. The drift speed $\boldsymbol{u}_{\text {front }}$ of wave front of the interchange instability during $14000 \lesssim \omega_{p e} t \lesssim 17000$ in Fig. 8 is estimated to be

$$
\boldsymbol{u}_{\text {front }} \simeq-\frac{0.14 \times 128 \Delta}{3000 \omega_{p e}^{-1}} \hat{e}_{y} \simeq-6.0 \times 10^{-3} \omega_{p e} \Delta \hat{e}_{y} .
$$

On the other hand, if $\boldsymbol{u}_{*}$ is assumed by ion diamagnetic drift velocity, it becomes

$\boldsymbol{u}_{*}=\frac{1}{e n} \frac{-c T_{i} \nabla n \times \boldsymbol{B}}{B^{2}} \simeq-\frac{1}{e \rho_{i}} \frac{c T_{i}}{B} \hat{e}_{y} \simeq-1.3 \times 10^{-1} \omega_{p e} \Delta \hat{e}_{y}$.

It is found $\left|\boldsymbol{u}_{*}\right| \gg\left|\boldsymbol{u}_{\text {front }}\right| \gg\left|\boldsymbol{u}_{\mathrm{g}}\right|$, although the wave front of the interchange instability drifts in the direction of the ion diamagnetic drift (that is, the gyroviscous cancellation phenomenon has been observed in this particle simulation with uniform magnetic field).

\section{SUMMARY}

The interchange instabilities in the geometry of Fig. 1 were investigated by using the electrostatic implicit PIC code. The growth rates of the interchange instability in the linearly growing phase in the particle simulation agree well with the theoretical linear growth rates with FLR which were obtained by solving Eq. (1). It is found that the interchange instability with the large FLR grows in two phases, that is, the linearly growing phase and the subsequent nonlinearly growing phase. The growth rate in the nonlinear phase is slower than that in the linear phase, and the interchange instability grows exponentially in both phases. The wave front of an interchange instability deviates from a sinusoidal shape in the nonlinear phase. The effects of gyroviscosity on the interchange instability seems to play an important role in its growth. The gyroviscous cancellation phenomenon has been observed in the particle simulation. The drift speed of wave front of the interchange instability, however, is much slower than the ion diamagnetic drift velocity.
The simulations were performed in the parameter of $k_{y}\left\langle\rho_{i}\right\rangle<1$, which has been assumed in all theoretical works with FLR. ${ }^{4,5,8,9,11,12,14}$ The particle simulation has introduced no approximations in the basic laws of mechanics and electricity. So the FLR is expected to stabilize the interchange instability completely in the real magnetically confined plasma. In the future, the particle simulations with $k_{y}\left\langle\rho_{i}\right\rangle \geqslant 1$ will be performed to research the FLR effects on an interchange instability in the range $\Omega_{c i} / \omega_{p i} \gg 1$.

${ }^{1}$ I. Katanuma, Y. Okuyama, and S. Kato, Nucl. Fusion 53, 043002 (2013).

${ }^{2}$ I. Katanuma, S. Sato, and Y. Okuyama, in 24th IAEA Fusion Energy Conference, San Diego, 2012, IAEA CN-197.

${ }^{3}$ R. D. Hazeltine and J. D. Meiss, Plasma Confinement (Dover Publications, Inc., Mineola, New York, 2003).

${ }^{4}$ M. N. Rosenbluth, N. A. Krall, and N. Rostoker, Nucl. Fusion Suppl. Part 1, 143 (1962).

${ }^{5}$ K. V. Roberts and J. B. Taylor, Phys. Rev. Lett. 8, 197 (1962).

${ }^{6}$ N. M. Ferraro and S. C. Jardin, Phys. Plasmas 13, 092101 (2006).

${ }^{7}$ P. Zhu, D. D. Schnack, F. Ebrahimi, E. G. Zweibel, M. Suzuki, C. C. Hegna, and C. R. Sovinec, Phys. Rev. Lett. 101, 085005 (2008).

${ }^{8}$ B. I. Cohen, R. P. Freis, and W. A. Newcomb, Phys. Fluids 29, 1558 (1986).

${ }^{9}$ B. Lane, R. S. Post, and J. Kesner, Nucl. Fusion 27, 277 (1987).

${ }^{10}$ M. W. Phillips and J. D. Callen, Phys. Fluids 27, 1733 (1984).

${ }^{11}$ V. P. Pastukhov and A. Yu. Sokolov, Fiz. Plasmy 17, 1043 (1991); Sov. J. Plasma Phys. 17, 603 (1991).

${ }^{12}$ Y. Sasagawa, I. Katanuma, Y. Mizoguchi, T. Cho, and V. P. Pastukhov, Phys. Plasmas 13, 122506 (2006).

${ }^{13}$ S. I. Braginskii, in Reviews of Plasma Physics, edited by A. M. A. Leontovich (Consultants Bureau, New York, 1965), Vol. 1.

${ }^{14}$ M. N. Rosenbluth and A. Simon, Phys. Fluids 8, 1300 (1965).

${ }^{15} \mathrm{~J}$. M. Dawson, in Methods in Computational Physics, edited by B. Alder (Academic Press, New York, 1970), Vol. 9.

${ }^{16}$ J. M. Dawson, H. Okuda, and B. Rosen, in Methods in Computational Physics, edited by B. Alder (Academic Press, New York, 1976), Vol. 16.

${ }^{17}$ H. Goede, D. G. Humanic, and J. M. Dawson, Phys. Fluids 26, 1812 (1983).

${ }^{18}$ P. L. Pritchett and F. V. Coroniti, J. Geophys. Res. 118, 146, doi:10.1029/2012JA018143 (2013).

${ }^{19}$ P. L. Pritchett, in Space Plasma Simulation, edited by J. Büchner, C. T. Dum, and M. Scholer (Springer, New York, 2012).

${ }^{20}$ B. I. Cohen, A. B. Langdon, and A. Friedman, J. Comput. Phys. 46, 15 (1982).

${ }^{21}$ B. I. Cohen, A. B. Langdon, and A. Friedman, J. Comput. Phys. 56, 51 (1984).

${ }^{22}$ A. B. Langdon, B. I. Cohen, and A. Friedman, J. Comput. Phys. 51, 107 (1983).

${ }^{23}$ T. H. Watanabe, Y. Todo, R. Horiuchi, K. Watanabe, and T. Sato, J. Comput. Phys. 127, 473 (1996).

${ }^{24}$ D. C. Barnes, T. Kamimura, J.-N. Leboeuf, and T. Tajima, J. Comput. Phys. 52, 480 (1983).

${ }^{25}$ For example, K. Miyamoto, Plasma Physics for Nuclear Fusion (MIT Press, Cambridge, MA, 1979).

${ }^{26}$ J. J. Ramos, Phys. Plasmas 12, 112301 (2005). 